\title{
Limited increase in antibody titers following mRNA SARS-CoV-2 vaccination for more than 3 years after final dose of anti-CD20 antibody
}

\author{
Yohei Funakoshi ${ }^{1}$ (1) Kimikazu Yakushijin ${ }^{1} \cdot$ Goh Ohji $^{2} \cdot$ Wataru Hojo $^{3} \cdot$ Hironori Sakai $^{3} \cdot$ Marika Watanabe $^{1}$. \\ Miki Saeki ${ }^{1}$ - Yuri Hirakawa ${ }^{1}$. Rina Sakai ${ }^{1}$. Sakuya Matsumoto ${ }^{1}$ - Yu Mizutani ${ }^{1}$. Akihito Kitao ${ }^{1}$ - Yoshiharu Miyata ${ }^{4}$. \\ Yasuyuki Saito ${ }^{5}$. Shinichiro Kawamoto ${ }^{6} \cdot$ Katsuya Yamamoto $^{1} \cdot$ Mitsuhiro Ito $^{7} \cdot$ Meiko Nishimura $^{1}$. \\ Yoshinori Imamura ${ }^{1} \cdot$ Naomi Kiyota $^{1,8} \cdot$ Hiroshi Matsuoka $^{4} \cdot$ Yasuko Mori $^{9} \cdot$ Hironobu Minami $^{1,8}$
}

Received: 6 September 2021 / Revised: 15 October 2021 / Accepted: 18 October 2021 / Published online: 4 January 2022

(c) Japanese Society of Hematology 2021

\begin{abstract}
We investigated the efficacy of BNT162b2 mRNA COVID-19 vaccine in patients with B-cell malignancies treated with anti-CD20 antibody. Although T-cell-mediated immune responses were detected even in patients receiving R-CHOP treatment, the S1 antibody titer following BNT162b2 vaccination remained only marginally increased for more than 3 years after the final dose of anti-CD20 antibody. We found no relationship between the percent of B-cells and S1 antibody titer. The duration of this suppression was much longer than we anticipated. Further protection and treatment strategies against COVID-19 for these patients are warranted.
\end{abstract}

Keywords SARS-CoV-2 vaccination $\cdot$ Anti-CD20 antibody $\cdot$ B-cell malignancies

Yohei Funakoshi and Kimikazu Yakushijin have contributed equally to this work.

Yohei Funakoshi

yohei@med.kobe-u.ac.jp

1 Division of Medical Oncology/Hematology, Department of Medicine, Kobe University Hospital and Graduate School of Medicine, Kobe, Japan

2 Division of Infection Disease Therapeutics, Department of Microbiology and Infectious Diseases, Kobe University Hospital and Graduate School of Medicine, Kobe, Japan

3 R\&D, Cellspect Co., Ltd, Morioka, Iwate, Japan

4 BioResource Center, Kobe University Hospital, Kobe, Japan

5 Division of Molecular and Cellular Signaling, Kobe University Graduate School of Medicine, Kobe, Japan

6 Department of Transfusion Medicine and Cell Therapy, Kobe University Hospital, Kobe, Japan

7 Division of Medical Biophysics, Kobe University Graduate School of Health Sciences, Kobe, Japan

8 Cancer Center, Kobe University Hospital, Kobe, Japan

9 Division of Clinical Virology, Center for Infectious Diseases, Kobe University Graduate School of Medicine, Kobe, Japan
Although the anti-CD20 antibodies rituximab and obinutuzumab are mainstay drugs against CD20-expressing hematological malignancies, they react with CD20 antigen expressed not only on malignant $\mathrm{B}$ cells but also on normal B cells. Accordingly, some recent reports have mentioned an increased risk of severe COVID-19 in patients treated with anti-CD20 antibodies [1-3]. Most recently, a few studies reported that anti-CD20 antibody impaired the efficacy of SARS-CoV-2 mRNA vaccination in triggering the humoral immune response [4-6]. In addition, the duration of the effect of anti-CD20 antibody on antibody production to BNT162b2 mRNA COVID-19 vaccine remains unclear. Furthermore, T-cell response after chemotherapy which includes anti-CD20 antibody is also unclear. Here, we prospectively evaluated the efficacy of the vaccine in patients with B-cell malignancies treated with anti-CD20 antibody. The study protocol was approved by Kobe University Hospital Ethics Committee (No. B2056714, 1481), and was conducted in accordance with the Declaration of Helsinki. All patients provided written informed consent to participate.

We first evaluated antibody titers in serum samples at 3 timepoints in 12 healthy volunteers (median age 75 years, range 57-82) and 3 patients with B-cell lymphoma 

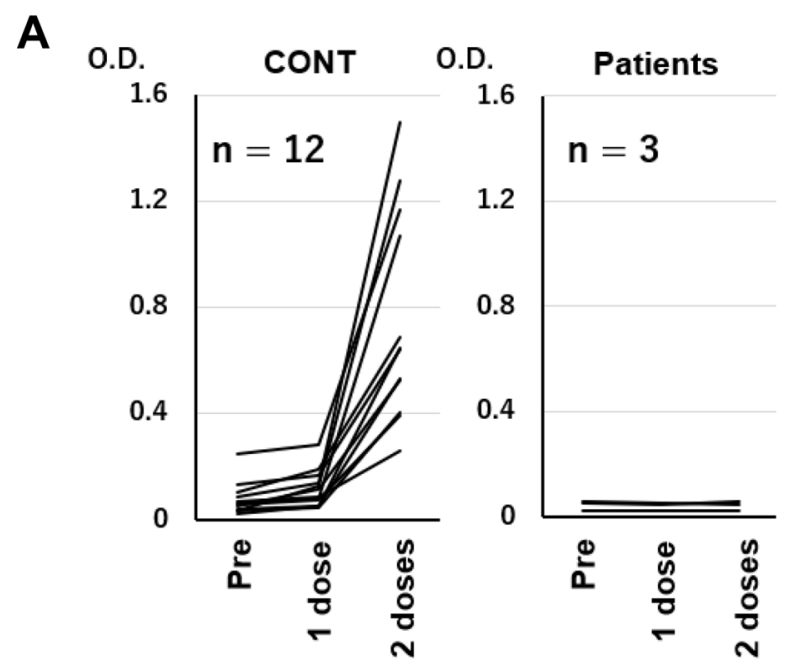

B

Patients



C



D



E

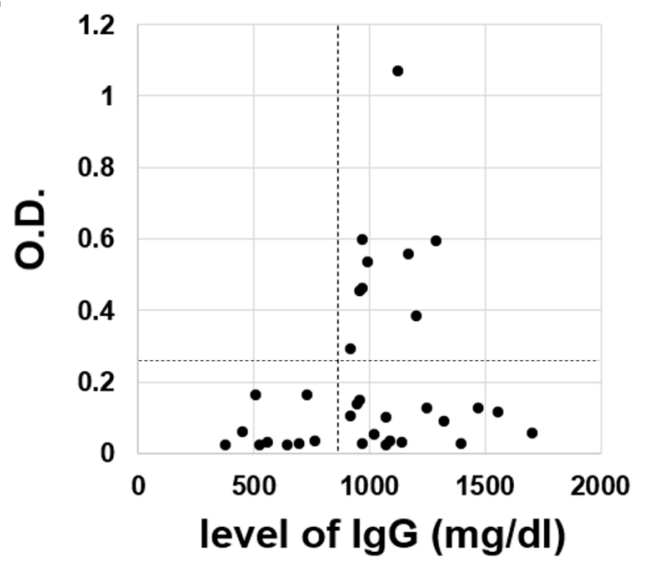


४Fig. 1 (A) Humoral quantitative anti-spike 1 (S1) antibody response at pre-vaccination (within 7 days prior to the first dose), 21 days ( \pm 3 days) after the first dose and 14 days ( \pm 7 days) after the second dose of BNT162b2 mRNA SARS-CoV-2 vaccine in healthy volunteers $(n=12)$ and diffuse large B-cell lymphoma patients treated with R-CHOP (rituximab, cyclophosphamide, doxorubicin, vincristine and prednisone) $(n=3)$. (B) Peripheral blood mononuclear cells obtained at pre-vaccination (within 7 days prior to the first dose) and 14 days ( \pm 7 days) after the second dose of BNT162b2 from Patients 1-3 were stimulated overnight with SARS-CoV-2 spike peptide for ex vivo assessment by IFN- $\gamma$ ELISPOT assay (human IFN- $\gamma$ SingleColor Enzymatic ELISPOT Kit, Cellular Technology Limited, USA) according to the manufacturer's protocol. (C) S1 antibody titers were measured 14 days ( \pm 7 days) after the second vaccination dose in 12 healthy donors and 36 patients who had received the final dose of anti-CD20 antibody 1-42 (median 17.5) months before vaccination. The horizontal dotted line indicates the lowest optical density (O.D.) value of S1 antibody titer in healthy donors. (D) Correlation between S1 antibody titer and percentage of CD19-positive B cells (normal range 6-23\%). (E) Correlation between S1 antibody titer and total $\mathrm{IgG}$ level. The vertical dotted line indicates the lower normal limit of serum IgG. The horizontal dotted line indicates the lowest optical density (O.D.) value of $\mathrm{S} 1$ antibody titer in healthy donors

undergoing R-CHOP therapy (age 73, 81, and 81 years) who had received 2 vaccine doses of BNT162b2, namely, at prevaccination, 21 days after the first vaccination, and 14 days after the second vaccination. IgG antibody titers for S1 protein were measured in serum samples with a QuaResearch COVID-19 Human IgM IgG ELISA kit (Spike Protein-S1) (Cellspect, Inc., Japan). Although titers in healthy control subjects were clearly increased, none of the three patients treated with R-CHOP developed antibodies even after the second vaccination (Fig. 1A). To determine SARS-CoV2 -specific T-cell reactivity in these three patients, we evaluated interferon (IFN)- $\gamma$ response to the SARS-CoV-2 spike peptide (PepTivator ${ }^{\circledR}$ SARS-CoV-2 Prot S1, Miltenyi Biotec, Germany) before and after vaccination with the ELISPOT assay (human IFN- $\gamma$ Single-Color Enzymatic ELISPOT Kit, Cellular Technology Limited, USA), and detected IFN- $\gamma$ responses after vaccination in all three patients (Fig. 1B).

Next, to investigate the duration of the effect of anti-CD20 antibody on antibody production to BNT162b2, we enrolled 36 patients (median age 74 years, range 50-87) who had received the final dose of anti-CD20 antibody 1-42 (median 17.5) months before vaccination (Table 1). S1 antibody titers were measured 14 days after the second dose of vaccination. Diagnoses included diffuse large B-cell lymphoma $(n=22)$, follicular lymphoma $(n=9)$, lymphoplasmacytic lymphoma/ Waldenström's macroglobulinemia $(n=3)$, and mantle cell lymphoma $(n=2)$. Thirty-four patients had received rituximab-based and 2 had received obinutuzumab-based therapy, with a median of 6 (range 3-20) courses. No patient had received any chemotherapy after the last anti-CD20 antibody dose. No patient who was vaccinated within 48 weeks of the last anti-CD20 antibody administration showed an increase in S1 antibody titers. Furthermore, titers in most patients were lower than the dotted line which indicates the lowest O.D. value of S1 antibody titer in healthy volunteers even among those vaccinated more than 3 years after the last administration (Fig. 1C). The proportion of patients above the dotted line was $11.1 \%$ (1/9) when the last dose was less than 1 year previously, 30.8\% (4/13) when 1-2 years previously, $37.5 \%$ (3/8) when 2-3 years previously and $33.3 \%$ (2/6) when more than 3 years previously. Finally, we investigated surrogate markers of antibody production ability. We found no relationship between the percentage of peripheral blood B-cells (CD19-positive cells) and S1 antibody titers (Fig. 1D). In terms of serum IgG level, although all patients $(n=9)$ with a total $\mathrm{IgG}$ level below the lower normal limit $(<870 \mathrm{mg} / \mathrm{dl})$ had low S1 antibody titers $(<0.26)$, below the lowest O.D. value in healthy donors, there was no correlation overall (Fig. 1E).

These findings indicate that the antibody-mediated response to vaccination in these patients following treatment with anti-CD20 antibody was considerably impaired for an extended time (range 1-42 months). A study group reported that vaccination efficacy was particularly impaired by anti-CD20 antibody in patients with chronic lymphocytic leukemia (CLL), who are in an immunosuppressive state due to both the inherent immune defect related to their primary disease and as a result of therapies, such as Bruton's tyrosine kinase inhibitor and anti-CD20 antibody [4]. In our study, we found that antibody production following vaccination with BNT162b2 was clearly suppressed for an extended period even without chemotherapy after the completion of anti-CD20 antibody administration in common B-cell malignancies, such as diffuse large B-cell lymphoma and follicular lymphoma. The duration of this suppression was much longer than we anticipated. Because most participants in this study were elderly, there is a possibility that the limited increases were related to age. Further study including younger participants is required. For patients with prolonged low titers, further protection and treatment strategies against COVID-19 warranted. We recommend that these patients continue to wear a face mask and wash their hands to prevent COVID-19 even after vaccination [7, 8]. Fortunately, T-cellmediated immune responses were detected even in patients during R-CHOP treatment with a combination therapy of anti-CD20 antibody and cytotoxic chemotherapy. However, it is necessary to clarify whether T-cell immunity is sufficient to protect patients against SARS-CoV-2. In terms of a COVID-19 treatment strategy for patients previously given anti-CD20 antibody treatment, anti-SARS-CoV-2 antibody therapeutics might be a good candidate [9].

Monitoring recovery of the ability to produce antibodies requires consideration. A study group examining a small case series $(n=5)$ of rituximab-treated patients with rheumatoid arthritis reported that a humoral immune response may be expected in the presence of circulating $B$ cells 
Table 1 Patient chracteristics

\begin{tabular}{ll}
\hline Total & $n=36$ \\
\hline Median Age (range, years) & $74(50-87)$ \\
Sex & 13 \\
Female & 23 \\
Male & \\
Diagnosis & 22 \\
DLBCL & 9 \\
FL & 3 \\
LPL/WM & 2 \\
MCL & \\
Anti-CD20 antibody & 34 \\
Rituximab-based & 2 \\
Obinutuzumab-based & $6(3-20)$ \\
Median course (range, courses) & \\
\hline
\end{tabular}

$D L B C L$ diffuse large B-cell lymphoma, $F L$ follicular lymphoma, $L P L / W M$ lymphoplasmacytic lymphoma/Waldenström's macroglobulinemia, $M C L$ mantle cell lymphoma

despite prior anti-CD20 antibody therapy [6]. However, our data revealed no relationship between $\mathrm{S} 1$ antibody titer and either the percentage $\left(r^{2}=0.033\right.$, Fig. 1E) or absolute number $\left(r^{2}=0.032\right.$, data not shown) of peripheral blood B cells. Two patients had high antibody titers (O.D., 0.56 and 0.38 ) even though their B-cell percentage was below the lower limit (normal range 6-23\%). We, therefore, considered that an increase in peripheral blood B-cell numbers does not necessarily mean that antibody production capacity following vaccination is recovering. Although a total $\operatorname{IgG}$ level below the lower normal limit may indicate impaired antibody production, recovery of $\mathrm{IgG}$ does not necessarily mean recovery of the capacity to produce antibody against SARS-CoV-2. Rather, monitoring this ability after vaccination might require the measurement of SARS-CoV-2 antibodies themselves.

Lower titers than those in healthy volunteers do not prove that there is no preventive effect of humoral immunity. Importantly, T-cell-mediated immune responses were detected even in patients during R-CHOP treatment. Therefore, we do not imply that vaccination is ineffective for these patients. We believe that it is important to continue to accumulate data and provide correct information for patients. At present, we recommend that these patients receive vaccination and continue to wear a face mask and wash their hands to prevent COVID-19.

In conclusion, we found that an increase in antibody titer following SARS-CoV-2 vaccination remains limited for more than 3 years after a final dose of anti-CD20 antibody. While, T-cell-mediated immune response was observed even during R-CHOP treatment.

\section{Declarations}

Conflict of interest KYak has received research grants and honoraria from Chugai Pharmaceutical. WH and HS are employed by Cellspect Co., Ltd. NK has received grants from Roche Phamaceuticals. HM has received research grants and honoraria from Chugai Pharmaceutical. The other authors declare no potential conflicts of interest.

\section{References}

1. PR Tepasse W Hafezi M Lutz J Kühn C Wilms R Wiewrodt 2020 Persisting SARS-CoV-2 viraemia after rituximab therapy: two cases with fatal outcome and a review of the literature Br J Haematol 190185188

2. P Guilpain $\mathrm{C}$ Bihan Le V Foulongne $\mathrm{P}$ Taourel $\mathrm{N}$ Pansu ATJ Maria 2021 Rituximab for granulomatosis with polyangiitis in the pandemic of covid-19: lessons from a case with severe pneumonia Ann Rheum Dis 80 e10

3. Schulze-Koops H, Krueger K, Vallbracht I, Hasseli R, Skapenko A. Increased risk for severe COVID-19 in patients with inflammatory rheumatic diseases treated with rituximab. Ann Rheum Dis. 2020.

4. Y Herishanu I Avivi A Aharon G Shefer S Levi Y Bronstein 2021 Efficacy of the BNT162b2 mRNA COVID-19 vaccine in patients with chronic lymphocytic leukemia Blood 13731653173

5. A Addeo PK Shah N Bordry RD Hudson B Albracht M Marco Di 2021 Immunogenicity of SARS-CoV-2 messenger RNA vaccines in patients with cancer Cancer Cell 391091 8.e2

6. Mrak D, Tobudic S, Koblischke M, Graninger M, Radner H, Sieghart D, et al. SARS-CoV-2 vaccination in rituximab-treated patients: B cells promote humoral immune responses in the presence of T-cell-mediated immunity. Ann Rheum Dis. 2021.

7. JT Brooks JC Butler 2021 Effectiveness of mask wearing to control community spread of SARS-CoV-2 JAMA 325998999

8. JPD Guidry NH O'Donnell LL Austin IA Coman J Adams PB Perrin 2021 Stay socially distant and wash your hands: using the health belief model to determine intent for COVID-19 preventive behaviors at the beginning of the pandemic Health Educ Behav 48424433

9. A Baum BO Fulton E Wloga R Copin KE Pascal V Russo 2020 Antibody cocktail to SARS-CoV-2 spike protein prevents rapid mutational escape seen with individual antibodies Science 369 10141018

Publisher's Note Springer Nature remains neutral with regard to jurisdictional claims in published maps and institutional affiliations. 